\title{
Hepatic NPC1L1 overexpression attenuates alcoholic autophagy in mice
}

\author{
YOULIN WANG ${ }^{1,2}$, PAN YANG $^{2-4}$, BO ZHANG $^{1,5}$, YAO DING $^{6}$, SHUN LEI $^{7}$, \\ YUNING HOU $^{2}$, XIAOQING GUAN ${ }^{2}$ and QINGWANG LI ${ }^{1}$
}

\begin{abstract}
${ }^{1}$ College of Animal Science and Technology, Northwest A\&F University, Yangling, Shaanxi 712100, P.R. China;
${ }^{2}$ Center for Molecular and Translational Medicine, Georgia State University, Atlanta, GA 30303, USA;

${ }^{3}$ Department of Cardiovascular Surgery, Chongqing General Hospital, University of Chinese Academy of Sciences,

Chongqing 400013; ${ }^{4}$ Emergency Department, The Second Affiliated Hospital of Chongqing Medical University,

Chongqing 400010; ${ }^{5}$ College of Basic Medical Sciences, Jiamusi University, Jiamusi, Heilongjiang 154007; ${ }^{6}$ Department of

Oncology, The Second Affiliated Hospital of Chongqing Medical University, Chongqing 400010; ${ }^{7}$ Department of Oncology,

Southwest Hospital, Third Military Medical University (Army Medical University), Chongqing 400038, P.R. China
\end{abstract}

Received January 29, 2019; Accepted June 27, 2019

DOI: $10.3892 / \mathrm{mmr} .2019 .10549$

\begin{abstract}
Alcohol consumption causes liver steatosis in humans. Metabolic disorders of lipids are one of the factors that cause liver steatosis in hepatocytes. Hepatic Niemann-Pick C1-like 1 (NPC1L1) regulates lipid homeostasis in mammals. The relationship between NPC1L1 and autophagy in those with a history of alcohol abuse is unclear. The present study aimed to investigate the function of NPC1L1 in the activation of hepatic autophagy in a mouse model with a human (h)NPC1L1 transgene under alcohol feeding conditions. The mice expressing hNPC1L1 (Ad-L1) or controls (Ad-null) were created by retro-orbital adenovirus injection. The Ad-L1 and Ad-null mice were fed with alcohol or a non-alcoholic diet to mimic chronic alcohol consumption in humans. Hepatic autophagy was demonstrated in isolated primary hepatocytes by monitoring autophagic vacuoles under fluorescence microscopy, and by western blotting for autophagic makers. Isolated hepatocytes from the livers of Ad-L1 mice were treated with different doses of ezetimibe to study the restoration of autophagy. Chronic alcohol feeding caused liver injury and steatosis, shown by significantly higher levels of plasma alanine transaminase and aspartate transaminase activity, and by hematoxylin and eosin staining in Ad-L1 and Ad-null mice. Compared to Ad-null control mice, the microtubule-associated proteins 1A/1B light chain 3 (LC3) particles in the isolated hepatocytes of Ad-L1 mice were decreased, both under alcohol
\end{abstract}

Correspondence to: Dr Qingwang Li, College of Animal Science and Technology, Northwest A\&F University, Building 5, 22 Xinong Road, Yangling, Shaanxi 712100, P.R. China

E-mail: liqingwangysu@aliyun.com

Key words: liver, Niemann-Pick C1-like 1, autophagy, chronic alcohol abuse, hepatocytes and non-alcoholic feeding. The ratio of LC3II/LC3I was significantly decreased, and the level of p62/sequestosome-1 protein was significantly increased in Ad-L1 mice compared with Ad-null mice after alcohol feeding. Levels of LC3II protein were statistically increased in hepatocytes isolated from Ad-L1 mice with ezetimibe treatment. The increase in LC3II expression was dose dependent. Within the tested range, it reached its highest level at $40 \mu \mathrm{M}$. The livers of Ad-L1 mice represent a more human-like state for the study of hepatic autophagy. Hepatic expression of human NPC1L1 resulted in an inhibition of autophagy; it may contribute to alcoholic fatty liver disease in humans.

\section{Introduction}

Those with a history of chronic alcohol abuse are at a higher risk of developing alcoholic fatty liver disease (AFLD), cirrhosis and, ultimately, liver failure (1). AFLD is represented by abnormal hepatic lipid accumulation in the early stages and steatohepatitis in the latter stages $(1,2)$. Lipids storage in lipid droplets is influenced by synthesis and degradation in the liver (3). Therefore, an increase in lipid degradation in lipid droplets via autophagy or lipolysis could ameliorate AFLD $(4,5)$.

Autophagy is a lysosome-dependent degradation process of intracellular organelles in mammalian cells. It is activated during stressful periods, such as energy deficiency (6), and is also influenced by cellular cholesterol levels and alcohol intake (6-8). The relationship between alcoholic beverages and autophagy is complex (9). Autophagy ameliorates liver steatosis in vivo $(5,10,11)$.

Alterations in cholesterol levels in cells can regulate autophagy $(8,12)$. By extension, the expression level of the cholesterol transporter Niemann-Pick C1-like 1 (NPC1L1) in the liver may also influence autophagy. NPC1L1 is a transmembrane cholesterol absorption transporter expressed in the liver and intestines in humans (13). The function of 
hepatic NPC1L1 is to prevent excessive cholesterol loss in humans $(14,15)$. By contrast, mouse NPC1L1 is expressed only in the intestines (16). The expression of human NPC1L1 in the mouse liver results in an elevation of the total plasma cholesterol level, and hepatic triglyceride secretion $(17,18)$. The hepatic cholesterol level is increased in the livers of apolipoprotein E-knockout mice with NPC1L1 overexpression (19). Furthermore, inhibition of NPC1L1 by ezetimibe can significantly activate autophagy in human hepatocytes (20). It is indicated that NPC1L1 may inhibit hepatic autophagy. In humans, NPC1L1 may be a potential factor contributing to alcoholic steatosis by reducing autophagy.

The present study assessed the function of NPC1L1 in the activation of hepatic autophagy in a mouse model expressing human NPC1L1 in the liver under conditions of alcohol consumption. It was concluded that NPC1L1 expression reduced hepatic autophagy.

\section{Materials and methods}

Generation of recombinant adenoviruses. cDNA of human NPC1L1 was cloned and NPC1L1 adenoviral vectors (Ad-L1) were constructed using the AdMax system (Hanbio Biotechnology Co., Ltd.) and purified through sucrose gradient ultracentrifugation (21). A control adenovirus (Ad-null) without encoded cDNA was also constructed. Adenoviral vectors were administered at a dose of $1 \times 10^{11}$ particles $\left(10.5 \times 10^{9}\right.$ plaque-forming units) via the retro-orbital vein into the mice. The sequences of the primers used to amplify human (h)NPC1L1 from the mouse liver for reverse transcription-quantitative PCR (RT-qPCR) are listed in Table I.

Animals and feeding. A total of 24 male mice (C57BL/6J, weighting 20-22 g) at the age of 8 weeks (Jackson Laboratory; stock no. 002207) were housed in standard cages at 4 mice were per cage. The conditions were $23^{\circ} \mathrm{C}, 50 \%$ humidity with a $12 \mathrm{~h}$ light/dark cycle and free access to water and a chow diet. A 10 day feeding plan was used to induce alcoholic liver steatosis during NPC1L1 expression (Fig. 1A). At the beginning, the mice were fed on a non-alcoholic liquid diet ad libitum (Bio-Serv; product no. F1259SP) for acclimatization. A total of 5 days later, 12 mice were changed to the Lieber-DeCarli alcoholic liquid diet (Bio-Serv; product no. F1258SP; $5 \mathrm{~g} / \mathrm{kg}$ body weight). The other 12 mice continued to be administered the non-alcoholic diet. After 3 days, Ad-L1 and Ad-null adenoviruses were injected retro-orbitally into 6 mice on the alcohol diet and 6 mice on the non-alcoholic diet. At the end of feeding, mice on the alcoholic diet were euthanized at $9 \mathrm{~h}$ after a single binge gavage (20\% alcohol). Instead of alcohol, the non-alcohol-fed mice were also euthanized at the same time after receiving a single dose of maltodextrin for energy balance. All mice were euthanized by $5 \%$ isoflurane (Hairui Chemical; cat. no. HR135327).

SDS-PAGE and western blot analysis. Liver tissues or primary hepatocytes were homogenized in the RIPA buffer (cat. no. 89900; Thermo Fisher Scientific, Inc.) followed by centrifugation at $12,000 \mathrm{x} \mathrm{g}$ for $10 \mathrm{~min}$ at $4^{\circ} \mathrm{C}$ to isolate the proteins for western blotting. Then, the protein concentrations were quantified by BCA protein assay kit (cat. no. 23227; Thermo Fisher
Scientific, Inc) via absorbance measurement using the LAS-4000 lumino-image analyzer (Fujifilm). Then, $30 \mu \mathrm{g}$ protein was loaded to each lane of $10 \%$ SDS-PAGE gel. Western blotting was performed following protocols described previously (17). Data were normalized against $\beta$-actin. The antibodies used in the present study were: Anti-microtubule-associated proteins 1A/1B light chain 3 (LC3) A/B antibody (cat. no. ab128025; Abcam); anti- $\beta$-actin antibody (cat. no. ab8227; Abcam); anti-p62/sequestosome-1 (SQSTM1) antibody (cat. no. ab91526; Abcam), anti-hNPC1L1 antibody (cat. no. ab124801; Abcam); anti-pan-cadherin antibody (cat. no. ab6528; Abcam); goat anti-mouse IgG (cat. no. ab6788; Abcam); and goat anti-rabbit IgG (cat. no. ab6720; Abcam). Primary antibodies were diluted as 1:3,000 in 5\% BSA for overnight incubation at $4^{\circ} \mathrm{C}$. Secondary antibodies were diluted as 1:10,000 in 5\% BSA for $1 \mathrm{~h}$ incubation at room temperature.

Duration of NPC1L1 expression. To determine how long the $\mathrm{hNPC1L1}$ expression lasted in the liver, a total of $10 \mathrm{C} 57 \mathrm{BL} / 6 \mathrm{~J}$ male mice received Ad-L1 injection, 2 mice were sacrificed at each time point $(0,1,3,7$ and 10 days) after injection and liver samples were harvested. Western blotting was performed using the aforementioned protocol and anti-hNPC1L1 antibody. The pan-cadherin antibody was used as a loading control.

Evaluation of liver injury by histopathology and biochemistry assay. Hematoxylin and eosin (H\&E) and Sirius red staining were performed to evaluate pathological changes in the liver. All specimens were fixed overnight at room temperature by $10 \%$ formalin and were embedded in paraffin wax. Paraffin were cleared from $5 \mu \mathrm{m}$ thick sections in three changes of xylene for $2 \mathrm{~min}$ per change at room temperature. The samples were hydrated at room temperature through three changes of $100 \%$ ethanol for 2 min per change followed by $95 \%$ ethanol and $70 \%$ ethanol for $2 \mathrm{~min}$, respectively. The slides were rinsed in running water at room temperature for $5 \mathrm{~min}$. The sections were stained in hematoxylin solution (cat. no. 143350, Shanghai Seebio Biotech, Inc.) for $3 \mathrm{~min}$ at room temperature, then rinsed under running water at room temperature for $10 \mathrm{~min}$. The sections were stained working eosin Y solution (cat. no. C0850110535, Nanjing reagent) for 2 min before dehydration by transfer from $95 \%$ ethanol (20 $\mathrm{min}$ ) to $100 \%$ ethanol ( 2 min each change), then three changes in xylene for 2 min per change. Slides were covered by glass coverslip with mounting medium (22). Picro-sirius red solution (cat. no. ab150681; Abcam) was using for $1 \mathrm{~h}$ staining at room temperature after de-waxing and hydration as described above Histological analyses were evaluated at x200 magnification using a Leica DM 2700P microscope (Leica Microsystems GmbH). Aspartate transaminase (AST) and alanine transaminase (ALT) were measured using an AST activity colorimetric assay kit (cat. no. K753; Bio-Vision, Inc.) and an ALT activity colorimetric assay kit (cat. no. K752; Bio-Vision, Inc.), respectively.

Primary hepatocyte isolation and fluorescence microscopy. Primary hepatocytes were isolated from non-alcohol-fed and alcohol-fed mice, as described previously (23). The hepatocytes were seeded onto collagen-coated coverslips in six-well plates. Cells were collected and fixed with $4 \%$ paraformaldehyde for $20 \mathrm{~min}$ at room temperature. LC3 protein was detected by 
Table I. Primer sequences.

Name $\quad$ Forward $\left(5^{\prime}-3^{\prime}\right)$

Reverse (3'-5')

Primer melting temperature $\left({ }^{\circ} \mathrm{C}\right)$

\begin{tabular}{llll}
\hline hNPC1L1 & AGAGTGAGCCTTACACAACCA & GCAGGACACGTTGGAGAGT & 60 \\
mAtg5 & TGTGCTTCGAGATGTGTGGTT & ACCAACGTCAAATAGCTGACTC & 60 \\
mAtg7 & TCTGGGAAGCCATAAAGTCAGG & GCGAAGGTCAGGAGCAGAA & 60 \\
mAtg12 & TGGCCTCGGAACAGTTGTTA & GGGCAAAGGACTGATTCACAT & 60 \\
GAPDH & GAGCCAAACGGGTCATCATC & CATCACGCCACAGCTTTCCA & 60 \\
\hline
\end{tabular}

immunofluorescence antibody (cat. no. ab128025; Abcam) as previously described. Briefly, slides were incubated for $10 \mathrm{~min}$ with PBS containing $0.1 \%$ Triton $\mathrm{X}-100$ then rinsed three times in PBS. The cells were incubated with $10 \%$ goat serum (cat. no. ab7481; Abcam) for $30 \mathrm{~min}$ at room temperature, then incubated cells in diluted primary antibody (1:100) in 1\% BSA in PBST overnight at $4^{\circ} \mathrm{C}$. Then, cells were incubated with goat anti-rabbit $\operatorname{IgG}(1: 1,000$, cat. no. ab150079; Abcam) at room temperature for $1 \mathrm{~h}$. The cell nucleus was stained with DAPI in Prolong Diamond Antifade Mountant (cat. no. P36962; Thermo Fisher Scientific, Inc.) while the slides were sealed. LC3 protein (red dot) in the cells was observed under a Leica DM2500 florescence microscope (magnification x1,000; Leica Microsystems $\mathrm{GmbH}$ ). Quantification was determined in 20 cells from captured images using Image $\mathrm{J}$ software (version 1.8.0, National Institutes of Health).

Ezetimibe treatment in cultured hepatocytes. To determine whether inhibition of NPC1L1 expression may restore the autophagy in alcohol-fed mice, primary hepatocytes were incubated in $5 \% \mathrm{CO}_{2}$ at $37^{\circ} \mathrm{C}$ ) in different concentrations of ezetimibe (0, 10, 20 and $40 \mu \mathrm{M}$; HR138776, Hairui Chemical, Co., Ltd.). After $1 \mathrm{~h}$, hepatocytes were collected for the protein expression of LC3 by western blotting.

$R T-q P C R$. RNA extraction from hepatocytes of Ad-L1 mice and Ad-null mice $(\mathrm{n}=6)$ were performed as described using a RNeasy Mini kit 250 (cat. no. 74106; Qiagen, Inc.). Purified RNA was transcribed at $42^{\circ} \mathrm{C}$ by QuantiTectRev. Transcription kit (cat. no. 205313; Qiagen $\mathrm{GmbH}$ ), and qPCR was performed using a SYBR ${ }^{\circledR}$ Green PCR Master Mix kit (cat. no. 4309155; Thermo Fisher Scientific, Inc.). Thermocycling conditions were $95^{\circ} \mathrm{C}$ for $2 \mathrm{~min}$, then 40 cycles of $95^{\circ} \mathrm{C}$ for $15 \mathrm{sec}$, annealing for $15 \mathrm{sec}, 72^{\circ} \mathrm{C}$ for $45 \mathrm{sec}$. After amplification, a melting curve was used to assess product purity. Annealing temperatures were optimized for each primer pair (Table I). GAPDH was used as an internal control and mRNA expression levels were calculated based on the $2^{-\Delta \Delta \mathrm{Cq}}$ method (24). mRNA levels for each gene represent the amount relative to that in control mice, which was arbitrarily standardized to 1. Sequences of the primers used for qPCR are given in Table I.

Statistical analysis. Data are presented as the mean \pm SEM of at least four independent experiments. Densitometric analysis was performed on the immunoblots using ImageJ 1.8.0 (National Institutes of Health). Statistical differences were determined using the Student's t-test and one-way
ANOVA (post hoc test: Tukey's multiple comparison test) or two-way ANOVA (post hoc test: Bonferroni). All statistics were performed using GraphPad Prism 5 (GraphPad Software, Inc.). $\mathrm{P}<0.05$ was considered to indicate a statistically significant difference.

Ethics statement. Experimental procedures and animal use and care protocols were in accordance with the guidelines of the Northwest A\&F University Institutional Committee for the Care and Use of Laboratory Animals, and were approved by the Committee on Ethical Use of Animals of Northwest A\&F University.

\section{Results}

Expression of human NPCILI (Ad-L1) in the mouse liver via adenovirus transfection. According to the experimental workflow (Fig. 1A), adenovirus-packaged human NPC1L1 cDNA was transfected retro-orbitally at day 3 after the commencement of the alcohol diet. NPC1L1 mRNA expression levels were evaluated in the mouse liver 3 days post-injection. The results revealed that mRNA expression in the Ad-L1 mice was 200-fold higher than that in the Ad-null mice (Fig. 1B; $\mathrm{P}<0.001)$. Western blot analysis showed that NPC1L1 protein was markedly expressed in the mouse liver starting from day 3 until day 7 after adenoviral injection (Fig. 1C), and was decreased at about 10 days post-injection.

The Ad-L1 mice behaved normally under the experimental conditions. The alcohol consumption and adenoviral treatment did not result in significant changes in body weight, liver weight or the liver/body weight ratio in Ad-null and Ad-L1 mice (Fig. 1D and E).

Lipids in the blood and liver were measured. Plasma total cholesterol was significantly increased in Ad-L1 mice regardless of alcohol feeding (Fig. 1F; $\mathrm{P}<0.05$ ). However, total hepatic cholesterol levels were similar under both non-alcoholic and alcohol feeding conditions (Fig. 1G). In contrast with the total cholesterol, total plasma triglyceride levels were similar between Ad-null and Ad-L1 mice (Fig. 1H) under both feeding states. Hepatic triglyceride levels were significantly higher in alcohol-fed mice in the Ad-L1 group (Fig. 1I; $\mathrm{P}<0.05$ ).

Chronic-binge alcohol uptake causes mild liver injury and steatosis in mice. A chronic-binge alcohol feeding plan was followed in the present study to induce alcoholic liver injury. Plasma AST and ALT activity were measured to evaluate liver 
A

Non-alcoholic liquid diet

Maltose dextrin

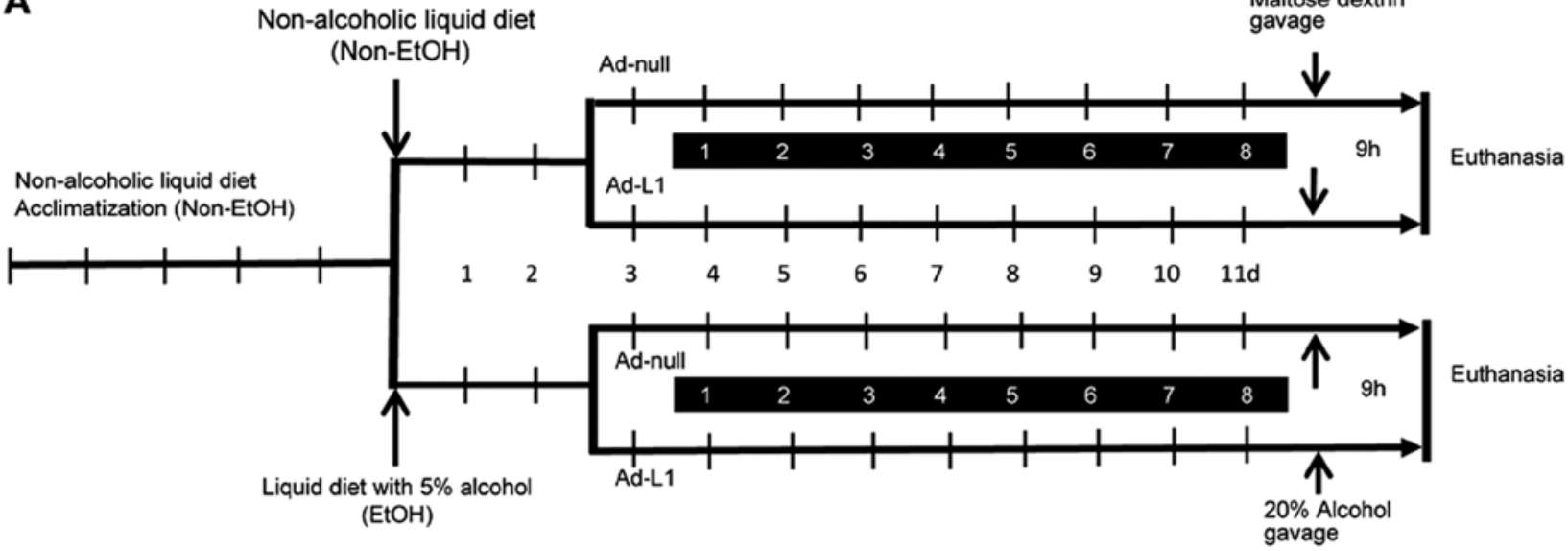

B

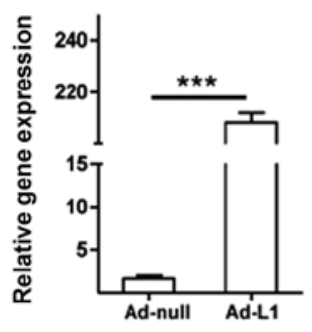

D

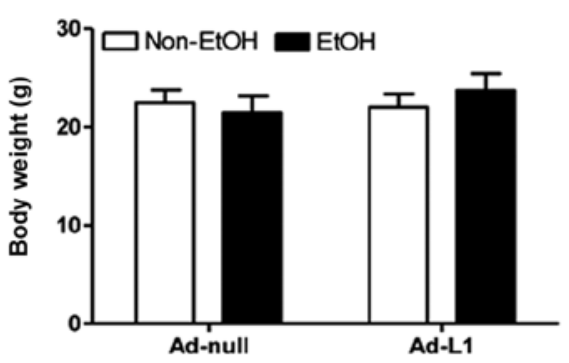

$\mathbf{F}$

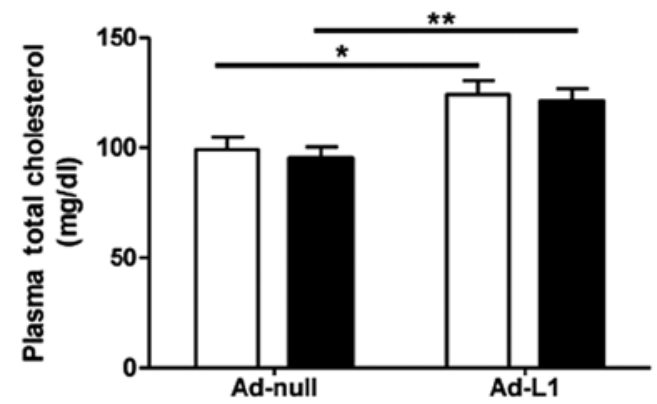

H

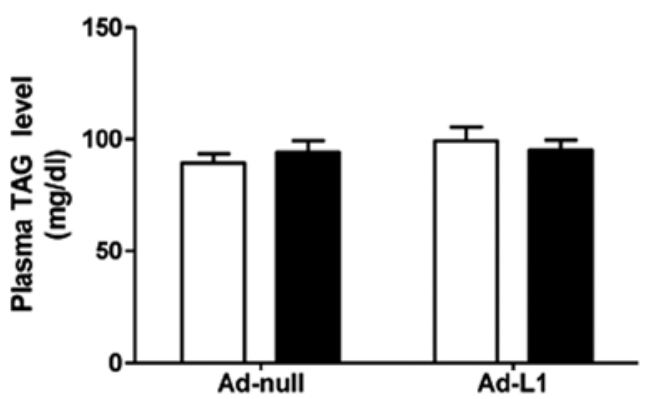

C

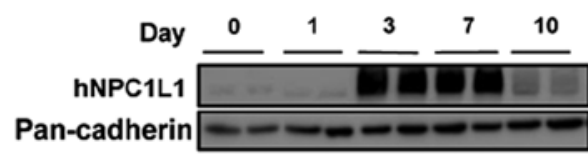

E

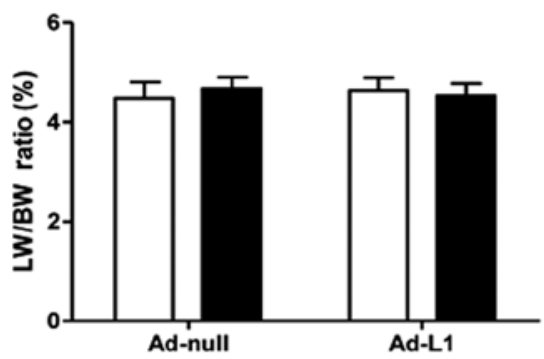

G

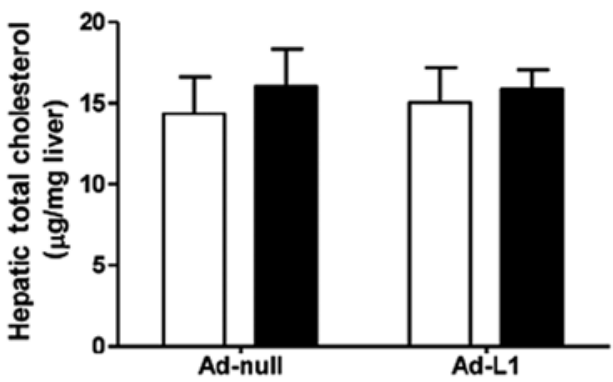

I

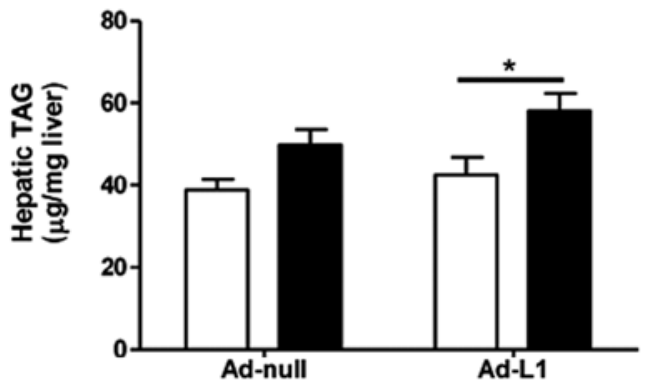

Figure 1. Creation and characterization of Ad-L1 and Ad-null mice. (A) Experimental workflow. (B) mRNA expression in the liver. (C) Protein expression in the livers of Ad-L1 mice. (D) Body weight comparison between EtOH and non-EtOH mice. (E) LW/BW ratio of EtOH mice compared with non-EtOH mice. (F) Plasma total cholesterol level. (G) Hepatic total cholesterol level. (H) Plasma TAG level. (I) Hepatic TAG level. Data in the graphs represent the mean \pm SEM. ${ }^{*} \mathrm{P}<0.05 ;{ }^{* *} \mathrm{P}<0.01 ;{ }^{* * *} \mathrm{P}<0.001$. TAG, triglyceride; EtOH, alcohol-fed; hNPC1L1, human Niemann-Pick C1-like 1; LW/BW, liver weight/body weight. 

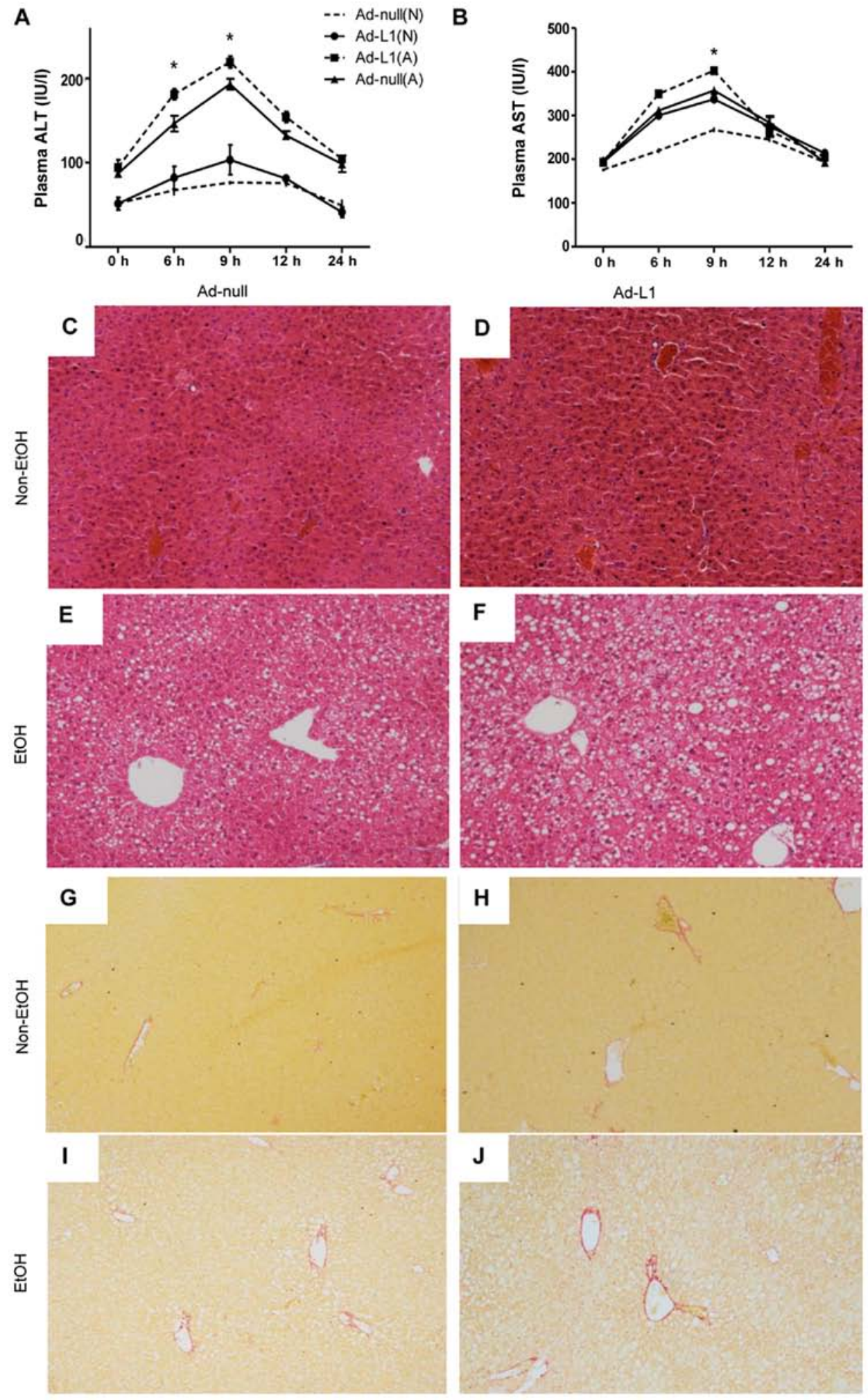

Figure 2. Alcohol feeding causes liver injury and steatosis. (A) Plasma ALT activity. (B) Plasma AST activity. Hematoxylin and eosin staining images of liver sections from (C) Ad-null and (D) Ad-L1mice fed with non- EtOH diet, and (E) Ad-null and (F) Ad-L1 mice treated with EtOH diet. Sirius red staining images of liver sections from (G) Ad-null and (H) Ad-L1 mice fed with non-EtOH diet and (I) Ad-null and (J) Ad-L1 mice fed with EtOH diet. Magnification, x200. ALT, alanine transaminase; AST, aspartate transaminase; EtOH, alcohol-fed. ${ }^{*} \mathrm{P}<0.05$.

injury. The results revealed that alcohol feeding caused increases in AST and ALT activity in the blood of Ad-L1 and Ad-null mice. Compared with the Ad-null mice after alcohol feeding, the Ad-L1 mice had significantly higher ALT activity (Fig. 2A; $\mathrm{P}<0.05)$. A small but significant difference was detected in plasma AST activity between Ad-L1 and Ad-null mice at $9 \mathrm{~h}$ after gavage (Fig. 2B; $\mathrm{P}<0.05$ ). This suggested that $9 \mathrm{~h}$ after the gavage was the appropriate time to evaluate liver injury.

Histologically, H\&E staining showed mild lipid accumulation in the livers of alcohol-fed mice (Fig. 2C-F). The lipid 

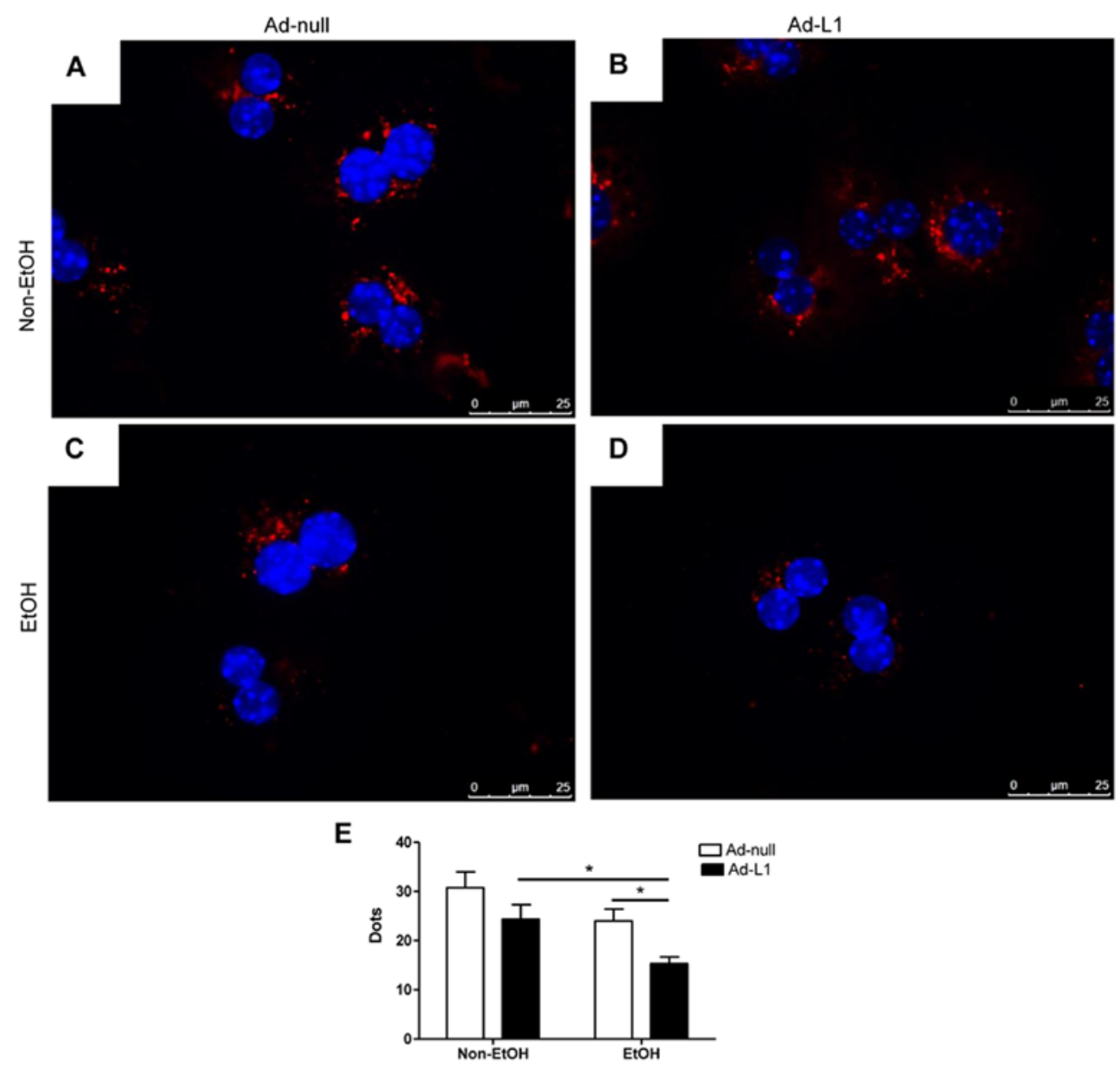

$\mathbf{F}$
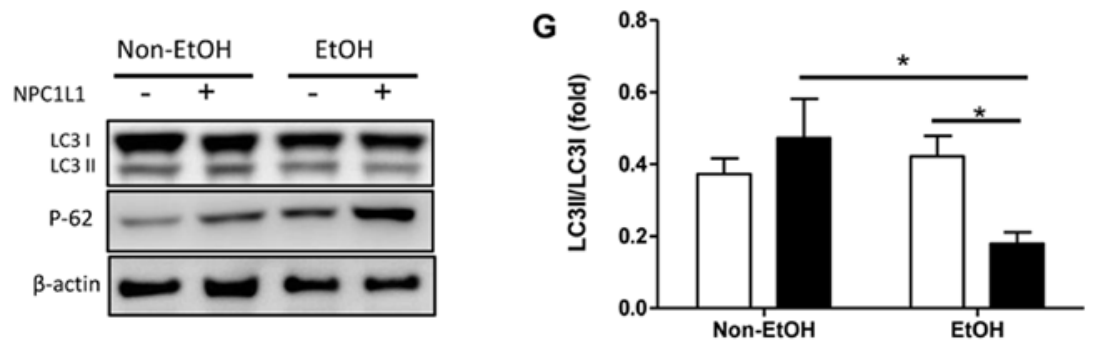

H

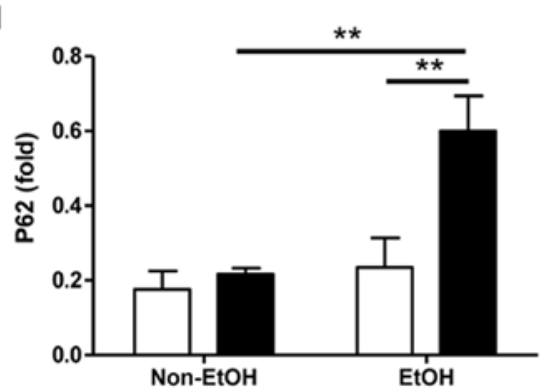

I

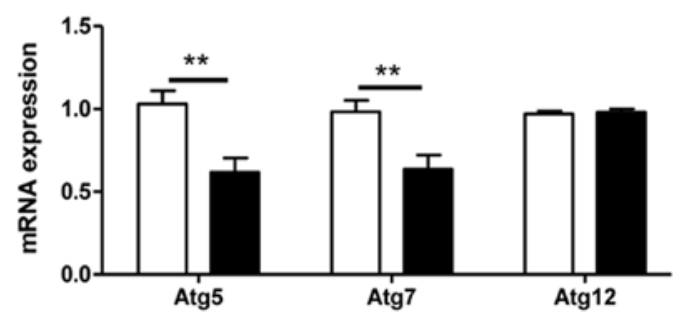

Figure 3. Autophagy is impaired in hepatocytes. Immunofluorescence images of isolated hepatocytes from (A) Ad-null and Ad-L1 (B) mice fed with fed with non-EtOH diet, and (C) Ad-null and (D) mice fed with EtOH diet. (E) Quantification of LC3 particles (red dots) in the fluorescence images. (F) Western blot analysis of LC3 and p62 proteins. (G) Densitometry of LC3 protein expression. (H) Densitometry of p62 protein expression. (I) Reverse transcription-quantitative PCR analysis of the mRNA expression of autophagy-related genes after alcohol feeding. Data in the graphs represent the mean \pm SEM. ${ }^{*} \mathrm{P}<0.05 ;{ }^{* *} \mathrm{P}<0.01$. LC3, microtubule-associated proteins 1A/1B light chain 3; EtOH, alcohol-fed; NPC1L1, Niemann-Pick C1-like 1.

accumulation was more pronounced in the livers of AD-L1 mice than that in Ad-null mice. This demonstrated that alcohol uptake induced mild liver steatosis. Sirius red staining showed no detectable fibrosis in the livers of alcohol-fed mice (Fig. 2G-J).
Autophagy is reduced in the hepatocytes of Ad-null mice after alcohol feeding. Fluorescence microscopy was used to monitor the formation of autophagic particles in isolated primary hepatocytes (Fig. 3A-D). Red dots indicate the intracellular autophagy marker LC3I/II, and blue dots stained with DAPI 
A
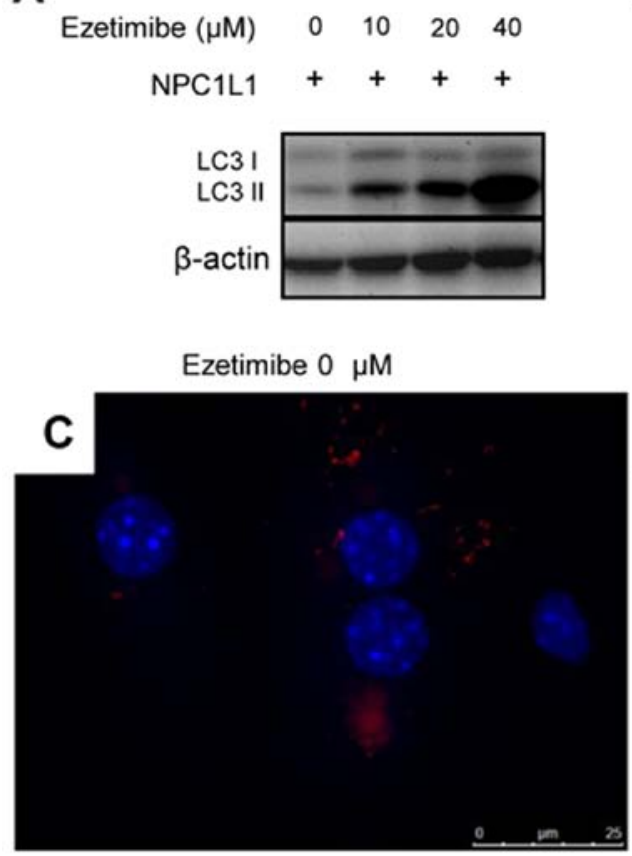

$\mathbf{E}$
B

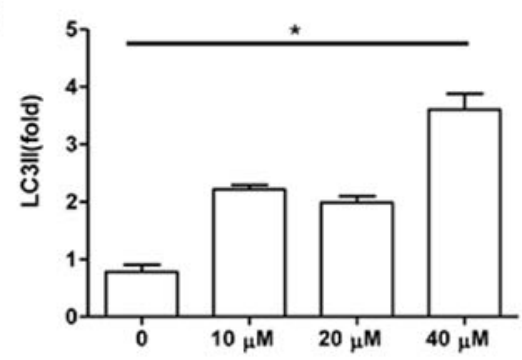

Ezetimibe $40 \mu \mathrm{M}$
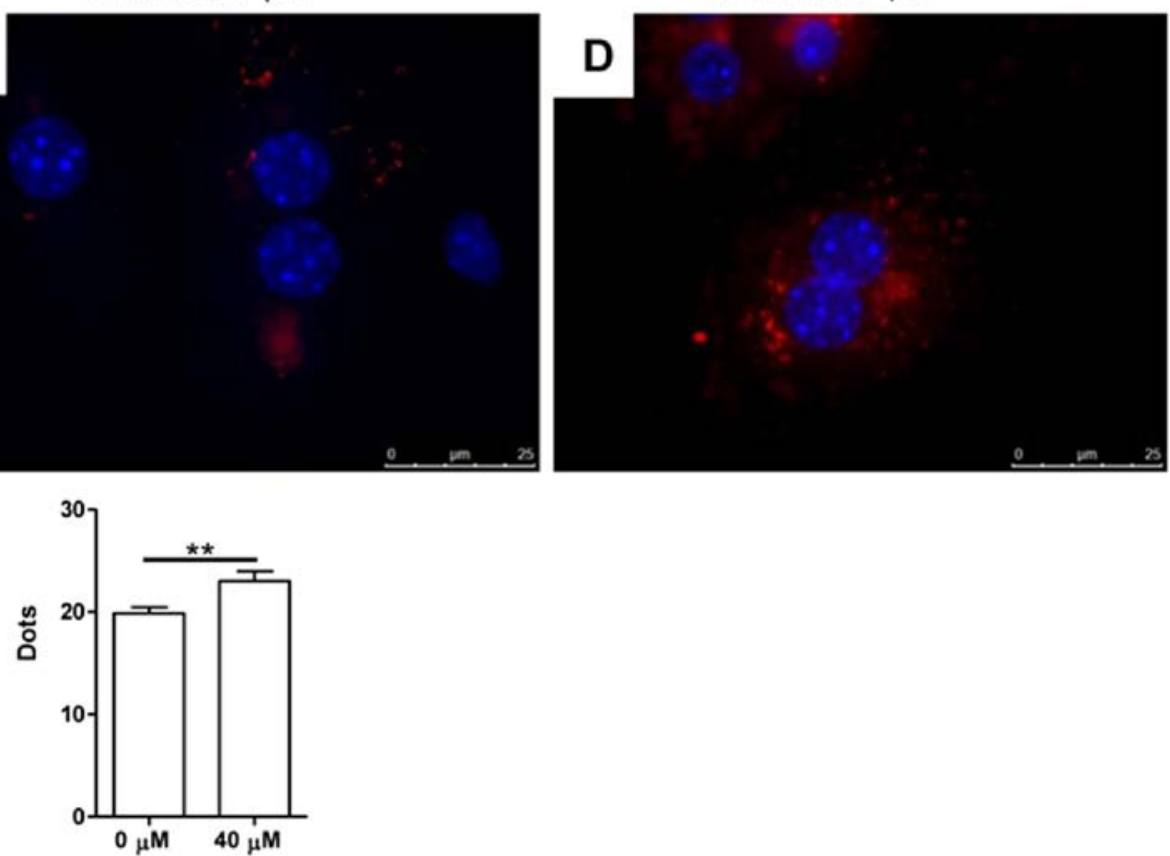

Figure 4. Ezetimibe activates autophagy in the hepatocytes of Ad-L1 mice after alcohol feeding. (A) Western blot analysis of LC3 expression in hepatocytes at different doses of ezetimibe. (B) Densitometry of LC3II expression. Immunofluorescence images with (C) no ezetimibe treatment and (D) treatment with $40 \mu \mathrm{M}$ ezetimibe. (E) Quantification of LC3 (red dots) in the images. Data in the graphs represent the mean $\pm \mathrm{SEM}$. $\mathrm{P}<0.05$; ${ }^{* *} \mathrm{P}<0.01$. LC3, microtubule-associated proteins 1A/1B light chain 3; NPC1L1, Niemann-Pick C1-like 1.

indicate the nuclei of the hepatocytes. Quantification of 20 hepatocytes from captured images (Fig. 3A-D) showed fewer red dots in the alcohol-fed mice (Fig. 3D and $\mathrm{E} ; \mathrm{P}<0.05$ ). The result was confirmed through analysis of the protein expression of the autophagy marker p62/SQSTM1 (Fig. 3F and H; $\mathrm{P}<0.01)$. The ratio of $\mathrm{LC} 3 \mathrm{II} / \mathrm{LCI}$ in the liver was unchanged in the alcohol-fed mice compared with the non-alcohol-fed mice.

Autophagy is attenuated in the hepatocytes of Ad-L1 mice. According to the immunofluorescence images, Ad-L1 mice had significantly less LC3 expression (Fig. 3D and E) compared with Ad-null mice under both alcoholic and non-alcoholic feeding conditions (Fig. 3B and E). These results were confirmed by western blot analysis (Fig. 3F). The LC3II expression was reduced. By contrast, p62/SQSTM1 protein expression was increased in Ad-L1 mice compared with Ad-null mice. This indicated that autophagy was inhibited. Quantification by densitometry showed that the LC3II/LC3I expression ratio (Fig. 3G) was significantly decreased and p62/SQSTM1 protein content (Fig. 3H) was significantly increased in Ad-L1 mice compared with Ad-null mice after alcohol feeding. The mRNA expression of autophagy-related markers atg5, atg7 and atg12 was evaluated after alcohol feeding in both Ad-L1 and Ad-null mice. The expression of atg5 and atg7, but not that of atg12, was significantly reduced in Ad-L1 mice compared with Ad-null mice after alcohol feeding (Fig. 3I; P<0.01). Together, these results indicated that NPC1L1 expression caused the inhibition of autophagy in the livers of mice after alcohol feeding.

Ezetimibe activates autophagy in alcohol-fed mouse hepatocytes. To further demonstrate that the reduction in autophagy was due to the NPC1L1 expression, primary hepatocytes from Ad-L1 mice were treated with different doses of ezetimibe, an inhibitor of NPC1L1 (Fig. 4A and B). The results indicated that LC3II protein expression was restored in Ad-L1 mice after ezetimibe treatment. The increase in the LC3II expression level was dose-dependent and reached its highest level at $40 \mu \mathrm{M}$ (Fig. 4A and $\mathrm{B} ; \mathrm{P}<0.05$ ). Quantification of the LC3 particles in fluorescence images indicated an increase at $40 \mu \mathrm{M}$ of ezetimibe (Fig. 4C-E; $\mathrm{P}<0.01$ ).

\section{Discussion}

Autophagy is the process of eliminating unnecessary or toxic intracellular components, including extra lipids, through 
activation of the lysosomal degradation process. Increased understanding of autophagy in hepatocytes after alcohol abuse could contribute to the treatment of AFLD (6). The effects of alcohol on hepatic autophagy have been studied in mouse models $(25,26)$. However, the findings from these studies are not entirely applicable to humans, because the expression of NPC1L1 is restricted to the intestines in rodents (27). The present study investigated hepatic autophagy in a more appropriate mouse model in which NPC1L1 was expressed in the liver, as it is in humans. It was also demonstrated that 10 day alcohol feeding plus a single binge gavage is an effective alcohol treatment procedure, because liver injury and steatosis were detected via elevation of blood AST and ALT activity, and by H\&E staining. This result was consistent with a previously reported binge model for AFLD $(28,29)$.

Alcohol treatment resulted in a reduction of hepatic autophagy in Ad-null mice, as supported by the results of the fluorescence microscopy in hepatocytes and liver p62 western blotting. The fluorescence microscopy images showed that there was a statistically significant reduction in LC3 in the hepatocytes of alcohol-fed mice compared with non-alcohol-fed mice. Moreover, p62 protein expression was also significantly increased in alcohol-fed mice. These results were in accordance with the consensus on alcoholic autophagy: That chronic alcohol treatment impairs autophagy (30), while acute alcohol treatment increases autophagy $(5,11)$.

In comparison to that in Ad-null mice, the expression of NPC1L1 in the liver led to a more pronounced reduction in autophagy in both the non-alcohol and alcohol feeding states. These results were confirmed by the expression of LC3 using fluorescence microscopy and western blotting. Other autophagic markers, p62/SQSTM1 protein expression and the mRNA level of atg5 and atg7, also indicated that hepatic autophagy was inhibited in the livers of Ad-L1 mice. The reduction in autophagy in primary hepatocytes of Ad-L1 mice could be restored by ezetimibe treatment. It was concluded that the expression of NPC1L1 impaired hepatic autophagy in Ad-L1 mice treated with chronic alcohol feeding.

A reasonable explanation for the autophagy inhibition mediated by NPC1L1 may involve changes in cellular cholesterol homeostasis. It is known that autophagy is highly sensitive to variations in cellular cholesterol homeostasis, and cholesterol-lowering drugs such as statins, cholestyramine and ezetimibe increase autophagy in hepatocytes (9). Hepatic NPC1L1 prevents cholesterol loss from bile in humans $(15,18)$. Overexpression of NPC1L1 in the liver results in free cholesterol absorption (30) and further impairs autolysosome clearance (9).

The experiments in human hepatocytes demonstrated that NPC1L1 was associated with the inhibition of autophagy, and ezetimibe treatment activated autophagy (15). To the best of our knowledge, this was the first in vivo study to show that overexpression of NPC1L1 in the liver impairs hepatic autophagy under alcohol feeding conditions. The findings of the present study suggested that activation of autophagy may be a potential therapy for AFLD via inhibition of NPC1L1. However, long-term investigation in other animal models, and clinical studies, are necessary before the treatment of AFLD patients with NPC1L1 inhibition.

\section{Acknowledgements}

Not applicable.

\section{Funding}

This study was funded by the National Natural Science Foundation of China (grant no. 31372280).

\section{Availability of data and materials}

All data generated or analyzed during this study are included in this published article.

\section{Authors' contributions}

YW,PY and QL made substantial contributions to the conception and design of the study. YW, PY, BZ, YD and SL were involved in data analysis and interpretation. $\mathrm{YH}$ and $\mathrm{XG}$ performed histological examination. YW, PY and QL drafted the manuscript and revised it critically for important intellectual content. All authors approved the final version of the manuscript and agreed to be accountable for all aspects of the work in ensuring that questions related to the accuracy or integrity of any part of the work are appropriately investigated and resolved.

\section{Ethics approval and consent to participate}

Experimental procedures and animal use and care protocols were in accordance with the guidelines of the Northwest A\&F University Institutional Committee for the Care and Use of Laboratory Animals, and were approved by the Committee on Ethical Use of Animals of Northwest A\&F University.

\section{Patient consent for publication}

Not applicable.

\section{Competing interests}

The authors declare that they have no competing interests.

\section{References}

1. Ishak KG, Zimmerman HJ and Ray MB: Alcoholic liver disease: Pathologic, pathogenetic and clinical aspects. Alcohol Clin Exp Res 15: 45-66, 1991.

2. Ramaiah S, Rivera $C$ and Arteel G: Early-phase alcoholic liver disease: An update on animal models, pathology, and pathogenesis. Int J Toxicol 23: 217-231, 2004.

3. Walther TC and Farese RV Jr: Lipid droplets and cellular lipid metabolism. Ann Rev Biochem 81: 687-714, 2012.

4. Lass A,Zimmermann R, Haemmerle G, Riederer M, Schoiswohl G, Schweiger M, Kienesberger P, Strauss JG, Gorkiewicz G and Zechner R: Adipose triglyceride lipase-mediated lipolysis of cellular fat stores is activated by CGI-58 and defective in chanarin-dorfman syndrome. Cell Metab 3: 309-319, 2006.

5. Ding WX, Li M, Chen X, Ni HM, Lin CW, Gao W, Lu B, Stolz DB, Clemens DL and Yin XM: Autophagy reduces acute ethanol-induced hepatotoxicity and steatosis in mice. Gastroenterology 139: 1740-1752, 2010.

6. Noh BK, Lee JK, Jun HJ, Lee JH, Jia Y, Hoang MH, Kim JW, Park KH and Lee SJ: Restoration of autophagy by puerarin in ethanol-treated hepatocytes via the activation of AMP-activated protein kinase. Biochem Biophys Res Commun 414: 361-366, 2011. 
7. Thomes PG, Ehlers RA, Trambly CS, Clemens DL, Fox HS, Tuma DJ and Donohue TM: Multilevel regulation of autophagosome content by ethanol oxidation in HepG2 cells. Autophagy 9: 63-73, 2013.

8. Singh R, Kaushik S, Wang Y, Xiang Y, Novak I, Komatsu M, Tanaka K, Cuervo AM and Czaja MJ: Autophagy regulates lipid metabolism. Nature 458: 1131-1135, 2009.

9. Li Y, Wang S, Ni HM, Huang H and Ding WX: Autophagy in alcohol-induced multiorgan injury: Mechanisms and potential therapeutic targets. Biomed Res Int 2014: 498491, 2014

10. Rasineni K, Donohue TM Jr, Thomes PG, Yang L, Tuma DJ, McNiven MA and Casey CA: Ethanol-induced steatosis involves impairment of lipophagy, associated with reduced Dynamin2 activity. Hepatol Commun 1: 501-512, 2017.

11. Ni HM, Du K, You M and Ding WX: Critical role of FoxO3a in alcohol-induced autophagy and hepatotoxicity. Am J Pathol 183: 1815-1825, 2013

12. Cheng J, Ohsaki Y, Tauchi-Sato K, Fujita A and Fujimoto T: Cholesterol depletion induces autophagy. Biochem Biophys Res Commun 351: 246-252, 2006.

13. Altmann SW, Davis HR, Zhu LJ, Yao X, Hoos LM, Tetzloff G, Iyer SP, Maguire M, Golovko A, Zeng M, et al: Niemann-Pick C1 Like 1 protein is critical for intestinal cholesterol absorption. Science 303: 1201-1204, 2004.

14. Davis HR Jr, Zhu LJ, Hoos LM, Tetzloff G, Maguire M, Liu J, Yao X, Iyer SP, Lam MH, Lund EG, et al: Niemann-Pick C1 Like 1 (NPC1L1) is the intestinal phytosterol and cholesterol transporter and a key modulator of whole-body cholesterol homeostasis. J Biol Chem 279: 33586-33592, 2004.

15. Temel RE, Tang W, Ma Y, Rudel LL, Willingham MC, Ioannou YA, Davies JP, Nilsson LM and Yu L: Hepatic Niemann-Pick C1-like 1 regulates biliary cholesterol concentration and is a target of ezetimibe. J Clin Invest 117: 1968-1978, 2007.

16. Davis HR Jr and Altmann SW: Niemann-Pick C1 like 1 (NPC1L1) an intestinal sterol transporter. Biochim Biophys Acta 1791: 679-683, 2009.

17. Wang Y, Tang W, Yang P, Shin H and Li Q: Hepatic NPC1L1 promotes hyperlipidemia in LDL receptor deficient mice. Biochem Biophys Res Commun 499: 626-633, 2018.

18. Tang W, Jia L, Ma Y, Xie P, Haywood J, Dawson PA, Li J and Yu L: Ezetimibe restores biliary cholesterol excretion in mice expressing Niemann-Pick C1-like 1 only in liver. Biochim Biophys Acta 1811: 549-555, 2011

19. Kurano M, Hara M, Tsuneyama K, Okamoto K, Iso-O N Matsushima T, Koike K and Tsukamoto K: Modulation of lipid metabolism with the overexpression of NPC1L1 in mouse liver J Lipid Res 53: 2275-2285, 2012.
20. Yamamura T, Ohsaki Y, Suzuki M, Shinohara Y, Tatematsu T, Cheng J, Okada M, Ohmiya N, Hirooka Y, Goto H and Fujimoto T: Inhibition of niemann-pick-type C1-likel by ezetimibe activates autophagy in human hepatocytes and reduces mutant $\alpha 1$-antitrypsin $\mathrm{Z}$ deposition. Hepatology 59: 1591-1599, 2014.

21. Simpson IA and Sonne O: A simple, rapid, and sensitive method for measuring protein concentration in subcellular membrane fractions prepared by sucrose density ultracentrifugation. Anal Biochem 119: 424-427, 1982.

22. Fischer AH, Jacobson KA, Rose J and Zeller R: Hematoxylin and eosin staining of tissue and cell sections. CSH Protoc 2008: pdb. prot4986, 2008.

23. Li WC, Ralphs KL and Tosh D: Isolation and culture of adult mouse hepatocytes. Methods Mol Biol 633: 185-196, 2010.

24. Livak KJ and Schmittgen TD: Analysis of relative gene expression data using real-time quantitative PCR and the 2(-Delta Delta $\mathrm{C}(\mathrm{T})$ ) method. Methods 25: 402-408, 2001

25. Ding W X, Manley S and Ni HM: The emerging role of autophagy in alcoholic liver disease. Exp Biol Med (Maywood) 236: 546-556, 2011.

26. Dolganiuc A, Thomes PG, Ding WX, Lemasters JJ and Donohue TM Jr: Autophagy in alcohol-induced liver diseases. Alcohol Clin Exp Res 36: 1301-1308, 2012.

27. Tang W, Ma Y and Yu L: Plasma cholesterol is hyperresponsive to statin in ABCG5/ABCG8 transgenic mice. Hepatology 44 1259-1266, 2006.

28. Ki SH, Park O, Zheng M, Morales-Ibanez O, Kolls JK, Bataller R and Gao B: Interleukin-22 treatment ameliorates alcoholic liver injury in a murine model of chronic-binge ethanol feeding: Role of signal transducer and activator of transcription 3 . Hepatology 52: 1291-1300, 2010.

29. Bertola A, Mathews S, Ki SH, Wang H and Gao B: Mouse model of chronic and binge ethanol feeding (the NIAAA model). Nat Protoc 8: 627-637, 2013

30. Betters JL and Yu L: NPC1L1 and cholesterol transport. FEBS Lett 584: 2740-2747, 2010.

This work is licensed under a Creative Commons Attribution-NonCommercial-NoDerivatives 4.0 International (CC BY-NC-ND 4.0) License. 